1. Vousden KH. p53: death star. Cell $2000 ; 103$ : 691-4.

2. Tyner SD, Venkatachalam S, Choi J, et al. p53 mutant mice that display early ageing-associated phenotypes. Nature 2002 ; 415: 45-3.

3. Homère. L'Odyssée. Chant XII.

4. Ferbeyre G, Lowe SW. The price of tumour suppression? Nature 2002 ; 415: 26-7.

5. Williams GC. Pleiotropy, natural selection and the evolution of senescence. Evolution 1957 ; 11 : 398-411

6. Guarente L, Kenyon C. Genetic pathways that regulate ageing in model organisms. Nature 2000 ; 408: 255-62

7. Tissenbaum HA, Guarente L. Increased dosage of a sir-2 gene extends lifespan in Caenorhabditis elegans. Nature 2001 ; 410 : 227-30.

8. Luo J, Nikolaev AY, Imai S, et al. Negative control of p53 by Sir2a promotes cell survival under stress. Cell 2001 ; 107 : 137-48.

9. Vaziri H, Dessain SK, Ng Eaton $\varepsilon$, et al. hSIR2 (SIRT1) functions as an NADdependent $\mathrm{p} 53$ deacetylase. Cell 2001 ; 107 : 149-59.

10. Derry WB, Putzke AP, Rothman JH. Caenorhabditis elegans p53: role in apoptosis, meiosis, and stress resistance. Science 2001 ; 294 : 591-5.
11. Migliaccio $\varepsilon$, Giorgio $M$, Mele $S$, et al. The $p 66^{\text {shc }}$ adaptor protein controls oxidative stress response and life span in mammals. Nature 1999 ; 402 : 309-13.

12. Ameisen JC. Le jeu de la vie avec la mort. Le suicide cellulaire joue-t-il un rôle dans le vieillissement des corps? La Recherche, Dossier Hors série « Le Temps », Avril $2001 ; 5$ : 76-9.

\title{
NOUVELLe
}

\section{Polymorphisme du gène codant pour le transporteur de la sérotonine et hypertension artérielle pulmonaire}

Saadia Eddahibi, Marc Humbert, Serge Adnot
> L'hypertension artérielle pulmonaire (HTAP) est une maladie fatale dans ses formes graves chez l'adulte ou chez le nouveau-né, dépourvue de traitement en dehors de la transplantation. L'élévation des résistances artérielles pulmonaires est liée à un remaniement hypertrophique de la paroi artérielle, associé à une réduction du lit vasculaire pulmonaire. La prolifération des cellules musculaires lisses vasculaires pulmonaires joue un rôle déterminant dans ces altérations. Des résultats obtenus récemment ont permis d'identifier le rôle déterminant du transporteur de la sérotonine (5-HTT) dans l'hyperplasie des cellules musculaires lisses vasculaires pulmonaires au cours de l'HTAP primitive humaine. Nous avons notamment incriminé un polymorphisme du gène codant pour le transporteur 5 -HTT dans la surexpression de la protéine et la prolifération des cellules musculaires lisses dans I'HTAP primitive humaine.
De nombreux arguments sont à l'origine de l'intéInserm U492 et porteur de la sérotonine ou 5-HTT [4]. Or, l'expression du 5-HTT est augmentée dans les cellules musculaires lisses d'artère pulmonaire Département de Physiologie, exposées à I'hypoxie [5], et Hôpital Henri Mondor, AP-HP, les souris déficientes en 551, avenue du Maréchal de Lattre de Tassigny, 94010 Créteil, France.

\section{serge.adnot}

@hmn.ap-hop-paris.fr

HTT sont protégées contre I'HTAP hypoxique. Ces observations nous ont permis de confirmer le rôle déterminant du 5-HTT au cours de I'HTAP expérimentale [5]. rêt actuel suscité par la sérotonine dans le développement de I'HTAP expérimentale et humaine. Plusieurs médicaments qui inhibent la recapture de la sérotonine en inhibant son transport, comme l'Aminorex dans les années 1960, ou la Dexfenfluramine dans les années 1990, ont été incriminés dans le développement de I'HTAP «primitive » chez I'homme [1]. Les données de la littérature ainsi que les observations faites dans notre laboratoire, en collaboration avec I'Unité Inserm 288, ont permis d'authentifier l'action déterminante de la sérotonine dans la physiopathologie de I'HTAP hypoxique. La sérotonine intervient par son action mitogénique sur les cellules musculaires lisses vasculaires [2-4]. Cet effet est dépendant de son accumulation intracellulaire et donc de l'activité et de l'expression du trans-
Une surexpression du 5-HTT est également associée à I'HTAP humaine. Elle est détectée dans les plaquettes de patients atteints d'HTAP primitive, ou surtout à partir de prélèvements de poumons obtenus à l'occasion de transplantations chez des patients atteints d'HTAP. Ces études montrent une augmentation de l'expression du 5-HTT dans les poumons, avec une localisation préférentielle et quasi exclusive du 5-HTT dans la média des vaisseaux, remaniés chez ces patients atteints d'HTAP primitive ou secondaire, avec une nette prédominance chez les patients souffrant d'une HTAP primitive (Figure 1). L'analyse in vitro de la réponse mitogénique de cellules musculaires lisses isolées d'artère pulmonaire normale, ou provenant de patients atteints d'HTAP, confirme l'activité mitogénique très marquée de la 
5-HT (5-hydroxytryptamine) sur les cellules musculaires lisses, action qui requiert le 5 -HTT, puisqu'elle est inhibée par le citalopram ou la paroxétine. L'activité du 5-HTT, estimée par la mesure de la capture de sérotonine tritiée dans les cellules musculaires lisses provenant de patients atteints d'HTAP primitive, est environ 2 à 3 fois supérieure à celle observée dans les cellules de sujets témoins. Les cellules musculaires lisses des patients atteints d'HTAP prolifèrent davantage en réponse à la sérotonine et au sérum que les cellules de sujets témoins. L'ajout d'un inhibiteur sélectif du 5-HTT supprime cette différence en rétablissant une activité proliférative identique dans les deux groupes de cellules. La prolifération des cellules en réponse à d'autres facteurs de croissance tels que le PDGF (platelet-derived growth factor), le TGF $\beta$, le FGFa (fibroblast growth factor acidic) ou l'EGF (epidermal growth factor) est par ailleurs comparable dans les deux groupes.

Ces résultats montrent que le 5 -HTT est surexprimé par les cellules musculaires lisses vasculaires pulmonaires de patients porteurs d'une HTAP primitive ou secondaire, et que cette surexpression est à l'origine d'une potentialisation de la réponse proliférative de ces cellules à la sérotonine, et donc au sérum, qui contient des concentrations élevées de sérotonine, de l'ordre de $1 \mathrm{mM}$.

Cette constatation et le fait que la surexpression du 5 -HTT dans des cellules musculaires lisses en culture qui persiste même dans les cellules isolées, nous a incité à rechercher une anomalie génétique ou un génotype particulier du 5-HTT chez ces patients [6].

L'analyse de la séquence promotrice du gène 5-HTT a révélé l'existence de deux génotypes qui diffèrent par le nombre de répétitions d'un motif en tandem («L» pour répétition en grand nombre, et «S» pour répétition en petit nombre) [7]. Ces variations alléliques de la séquence promotrice du gène, $\mathrm{L}$ ou $S$, déterminent respectivement une vitesse de transcription élevée ou basse. Nous avons montré que l'expression du 5 -HTT varie en fonction du génotype $S / S, L / S$, ou $L / L$, dans des cellules musculaires lisses d'artère pulmonaire de sujets sains et détermine la réponse proliférative des cellules à la 5 -HT ou au sérum. Nous avons donc recherché un lien entre l'augmentation du 5-HTT dans I'HTAP primitive ou secondaire et la fréquence de l'allèle «L»du promoteur du gène codant pour cette protéine. Des résultats obtenus chez 89 patients porteurs d'une HTAP primitive montrent que la situation homozygote $\mathrm{L} / \mathrm{L}$ est présente chez $65 \%$ des patients alors qu'elle ne dépasse pas $27 \%$ dans la population générale. La situation homozygote $L / L$ pourrait donc représenter un facteur prédisposant au développement d'une HTAP primitive en favorisant l'hyperpla-
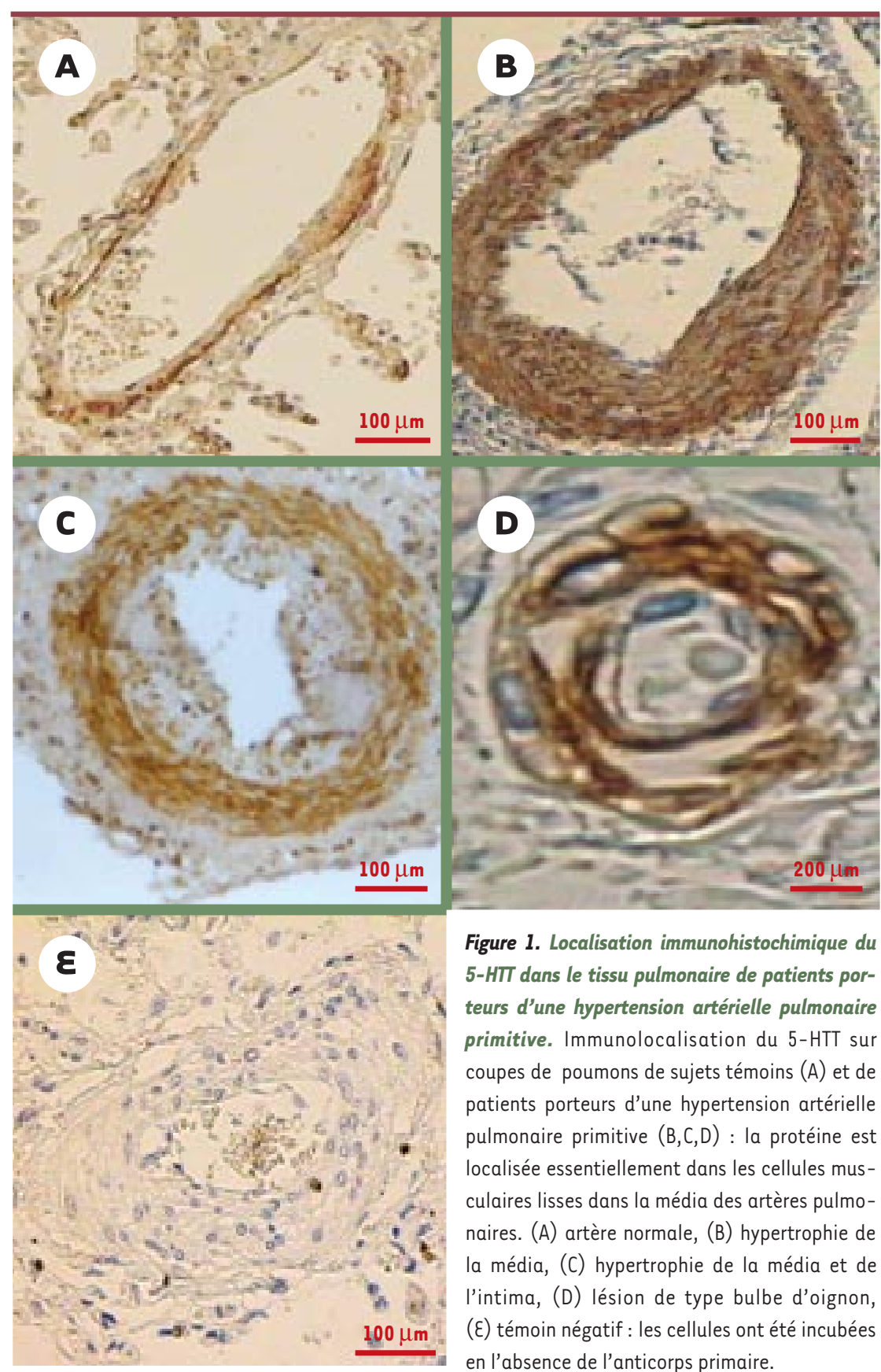

Figure 1. Localisation immunohistochimique $d u$ 5-HTT dans le tissu pulmonaire de patients porteurs d'une hypertension artérielle pulmonaire primitive. Immunolocalisation du 5-HTT sur coupes de poumons de sujets témoins ( $A$ ) et de patients porteurs d'une hypertension artérielle pulmonaire primitive $(B, C, D)$ : la protéine est localisée essentiellement dans les cellules musculaires lisses dans la média des artères pulmonaires. (A) artère normale, (B) hypertrophie de la média, (C) hypertrophie de la média et de l'intima, (D) lésion de type bulbe d'oignon, $(\varepsilon)$ témoin négatif : les cellules ont été incubées en l'absence de l'anticorps primaire. 
sie des cellules musculaires lisses vasculaires pulmonaires via le 5-HTT. Les cellules des patients de génotype L/L ont cependant une activité proliférative plus importante et une expression plus marquée du 5-HTT que celles de sujets témoins de même génotype. II semble donc que les différences d'expression du 5-HTT entre patients et témoins, à l'origine d'une augmentation de l'activité proliférative des cellules musculaires lisses des patients, ne puissent pas entièrement être expliquées par le polymorphisme du promoteur du gène.

L'aide diagnostique apportée par la caractérisation allélique du promoteur du gène du 5 -HTT peut être très utile pour la détection de patients à risque dans une population exposée au développement d'une HTAP. En effet, l'association du génotype $L / L$ au développement de I'HTAP pourrait s'appliquer non seulement à I'HTAP primitive mais aussi à toute forme d'HTAP sévère développée au cours de maladies telles que les collagé- noses, l'hypertension portale, les cardiopathies comportant un shunt, I'HTAP persistante du nouveau-né, la maladie thrombo-embolique, l'insuffisance respiratoire chronique, l'exposition à l'altitude, etc. Des études complémentaires pourront être réalisées dans ces affections. Les implications thérapeutiques sont potentiellement considérables. Les résultats obtenus chez l'animal montrent en effet que certains inhibiteurs très sélectifs du 5-HTT atténuent I'HTAP hypoxique. La reconnaissance d'une surexpression du 5 - HTT d'origine génétique chez les patients porteurs d'une HTAP incite à évaluer l'action thérapeutique potentielle de tels médicaments. $\diamond$
1. MacLean M, Herve P, Eddahibi S, Adnot $S$. 5-hydroxytryptamine and the pulmonary circulation: receptors, transporters and relevance to pulmonary hypertension. BrJ Pharmacol 2000 ; 131 : 161-8.

2. Eddahibi S, Raffestin B, Pham I, et al. Treatment with 5-hydroxytryptamine potentiates development of pulmonary hypertension in chronically hypoxic rats. Am J Physiol 1997 ; 272 : H1173-81.

3. Eddahibi S, Raffestin B, Launay JM, Sitbon M, Adnot S. Effect of dexfenfluramine treatment in rats exposed to acute and chronic hypoxia. Am J Respir Crit Care Med 1998 ; 157 : 1111-9.

4. Eddahibi S, Fabre V, Boni C, et al. Induction of serotonin-transporter by hypoxia in pulmonary vascular smooth muscle cells: relationship with the mitogenic action of serotonin. Circ Res 1999 ; $84: 329-36$.

5. Eddahibi $S$, Hanoun $N$, Frisdal $\varepsilon$, et al. Attenuated hypoxic pulmonary hypertension in mice lacking the 5hydroxytryptamine transporter gene. J Clin Invest 2000; 105 : 1555-62.

6. Eddahibi S, Humbert M, Fadel $\varepsilon$, et al. Serotonin transporter overexpression is responsible for pulmonary artery smooth muscle hyperplasia in primary pulmonary hypertension. J Clin Invest 2001 ; 108 : 1141-50.

7. Lesch KP, Bengel D, Heils A, et al. Association of anxiety-related traits with a polymorphism in the serotonin transporter gene regulatory region. Science $1996 ; 274$ : 1527-31.

\section{NOUVELLE}

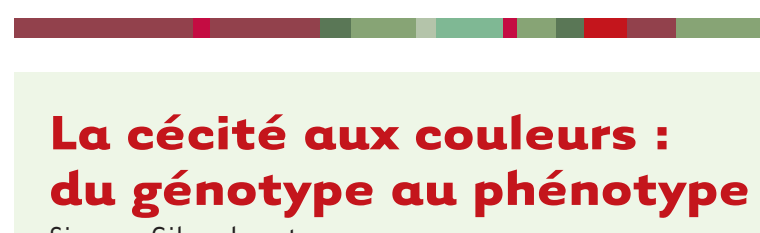

Simone Gilgenkrantz

$>$ Les troubles de la vision des couleurs sont fréquents chez les sujets masculins dans l'espèce humaine car les gènes codant pour les opsines rouges et vertes sont situés en Xq28. Jean-Claude Dreyfus avait, en son temps, parfaitement décrit les bases moléculaires de la vision $(\rightarrow) \mathrm{m} / \mathrm{s}$ 1987, des couleurs et du daltonisme $(\rightarrow)$. Ce 3 (suppl 7): terme de daltonisme se perd, mais il 20-22

est bon de se souvenir que John Dalton avait publié son trouble de la vision en 1794 à la Société philosophique l'on put vérifier enfin, par analyse de son ADN, que John Dalton était deutéranope $(\rightarrow)$.

et littéraire de Manchester, et avait demandé que ses yeux soient disséqués après sa mort. Ce n'est qu'en 1995 que

$\rightarrow$ m/s $1995, n^{\circ} 5$, p. 769 Rappelons que plusieurs gènes $R$ (rouge) et $G$ (vert), au nombre de trois ou quatre, sont disposés en série, l'un derrière l'autre, mais que seuls les deux premiers gènes de la série sont exprimés
9, rue Basse, 54330

Clerey-sur-Brenon, France.

et déterminent la vision des couleurs. En raison d'un degré important d'homologie, et de leur disposition en tandem, ces gènes ont tendance à faire des crossing-over inégaux. II en résulte des gènes hybrides $R / G$ ou $G / R$ qui affectent la production des pigments localisés dans la membrane plasmique des cellules photoréceptrices (cônes) de la rétine. La perte de fonction du gène $R$ (situé en 5' de la série de 3 ou 4 gènes) entraîne une perte de la vision du rouge 